\title{
Molecular diagnosis of tuberculosis: the need for new diagnostic tools
}

The resurgence of tuberculosis over the past decade is now a familiar story to readers of Thorax. The interaction between HIV and mycobacteria is one of the significant causes. The consequences of this interaction have been well described and have served to exacerbate the problem of confirming a diagnosis of tuberculosis.

Throughout the developing world the majority of adults are infected with Mycobacterium tuberculosis which has led to tuberculosis being seen as one of the commonest secondary diseases associated with HIV in these societies. ${ }^{12}$ In contrast, disease due to Mycobacterium avium is rarely seen, which has allowed sputum microscopy to retain its specificity as a diagnostic tool.

Unlike many of the organisms that afflict the HIV seropositive patient, $M$ tuberculosis is a virulent pathogen and therefore causes disease in those with minimal immunosuppression who are infected with HIV. These patients present with features indistinguishable from those seen before the HIV era. As immunosuppression deepens, tuberculosis becomes an increasingly disseminated disease and provokes a less brisk immune response. Cavitation becomes rarer, lesions are less confined to the apices, and extrapulmonary disease becomes more common. ${ }^{34} \mathrm{~A}$ consequence of these changes is that fewer patients have positive sputum smears on direct microscopy, which accords with the smaller numbers of bacilli found in sputum from HIV seropositive patients. ${ }^{5-7}$

HIV-related immunosuppression also leads to an increased susceptibility to disease following recent infection or reinfection. ${ }^{8}$ This may also explain the increase in numbers of cases with disease reminiscent of primary infection with pleural effusions, hilar lymphadenopathy and miliary spread, most of whom have negative sputum microscopy.

In Lusaka, the capital city of Zambia, for instance, $75 \%$ of tuberculosis patients are HIV seropositive. ${ }^{9}$ In 1993 more than one third of pretreatment samples that grew $M$ tuberculosis were negative on direct smear (G Kahenya, Chest Disease Laboratory, Lusaka, Annual Report 1993) and only $19 \%$ of new cases were smear positive (R Msiska, National AIDS, STD, TB and Leprosy Control Programme, Annual Report 1993), although the latter statistic may also reflect poor utilisation of the microscopy services.

From a theoretical viewpoint the rise in smear negative disease does not alter the approach to tuberculosis control. The goal must still be to find smear positive cases and treat them effectively. However, it is clear that the large number of patients with tuberculosis but negative sputum smears will be a huge stress on the diagnostic and clinical services in countries where both epidemics overlap, and a new diagnostic tool that is more rapid than culture and more sensitive than microscopy, but still specific, would be a major advance.

In contrast, in the industrialised world mycobacterial disease in those infected with HIV is more frequently due to $M$ avium, particularly in those with advanced immunosuppression, and the priority here is the individual patient rather than the community. In addition to the need for rapid diagnosis in the paucibacillary case, there is also a real need to distinguish between different acid fast bacilli and traditional methods involving cultivation may take weeks.

\section{Approaches through molecular genetics}

Although mycobacteria raise technical and safety issues when brought into laboratories, the recent advances that have been made in mycobacterial molecular genetics have led to better understanding of pathogenesis and epidemiology, as well as to possibilities for diagnosis and avenues to explore for vaccine development. ${ }^{1011}$

In the diagnostic field detection of nucleic acids, either directly by hybridisation to a labelled probe or following amplification of specific sequences of DNA or RNA, raises the exciting possibility of a sensitive specific diagnosis that would provide an answer within hours rather than days or weeks.

As particular genes are cloned and sequenced, it becomes possible to select target sequences that are specific to species in the $M$ tuberculosis complex. Such sequences have been used as probes to try to detect small amounts of tuberculous DNA in clinical material. ${ }^{12}$ Sadly, the sensitivity has been disappointing and the only commercial probe to be released was withdrawn when it was realised that it was little better than microscopy, despite considerably more effort and expense.

\section{Molecular amplification techniques}

The description of the polymerase chain reaction (PCR) using a thermostable DNA polymerase to amplify DNA exponentially by simply cycling the temperature of the reaction ${ }^{13}$ has led to a flurry of activity to produce diagnostic tests for tuberculosis. In principle, the exquisite sensitivity of PCR, combined with the inherent specificity of the target DNA sequence, makes it an attractive option. The first paper to describe PCR as a diagnostic test for tuberculosis appeared in $1989,{ }^{14}$ and since then there have been over 60 similar publications attesting to the perceived importance of new diagnostic tools, but also highlighting the difficulties experienced in taking the technique from the research bench into the routine laboratory. Nor is PCR the only amplification technique being developed with commercial backers. Ligase chain reaction (LCR), ${ }^{15}$ transcription mediated amplification (TMA) (Gen-probe "Amplified $M$ tuberculosis Direct"), ${ }^{1617}$ and strand displacement amplification (SDA) ${ }^{18}$ are all capable of exponential amplification of nucleic acids from a specific target, either by cycling the temperature (PCR, LCR) or by the dynamics of specific enzymes at a constant temperature (TMA, SDA). The limiting steps are likely to be similar for all these techniques - namely, how to prevent contamination of reactions with the products of previous assays and how best to extract the mycobacterial nucleic 
acid from clinical material in a form that is easily amplified.

False positive results will usually be caused by contamination occurring at some stage between the collection of the sample and the analysis of the amplified product. That contamination with live organisms occurs in routine settings has been known for a long time ${ }^{19}$ and reconfirmed by molecular techniques. ${ }^{20}$ However, all the amplification techniques are particularly susceptible to "carryover" problems where infinitesimal amounts of the product of previous reactions are allowed to get into the reactions being prepared by contaminating reagents, pipettes, laboratory staff's clothing or skin, or through aerosols produced by opening the lids of reaction tubes. The scale of the problem was demonstrated in a comparison of seven different laboratories, all of whom had already developed PCRbased assays with a view to diagnosing tuberculosis. ${ }^{21}$ In four of these laboratories specificity was less than $80 \%$, and in the remaining three the sensitivity was rather disappointing with only $60 \%$ of the samples with $5000 \mathrm{M}$ bovis BCG cells $/ \mathrm{ml}$ being detected, although the target sequence is usually present in 5-20 times the concentration in pathogenic $M$ tuberculosis isolates.

Measures to prevent carryover contamination include strict physical separation of amplified product from the area where reactions are being set up, with dedicated equipment and no access to staff coming from the "contaminated area". ${ }^{22}$ A three room system adds additional security by preventing any DNA from entering the laboratory in which stock solutions are prepared and opened. ${ }^{23}$ Incorporating deoxyuracil triphosphate into the reaction mixture in the place of deoxythymidine triphosphate means that the amplified product can be rendered unamplifiable by uracil-N-glycosylase at the start of each reaction, leaving genuine target DNA unaffected. ${ }^{24}$ Despite these precautions, some false positive results will continue to occur so that all samples should be processed in duplicate and only considered positive if both duplicates contain product.

"Nested" PCR involves amplifying DNA directly from a clinical sample and then taking a small aliquot of the resulting products and re-amplifying them using primers internal to the first set on the same target. ${ }^{25}$ The advantages are that any inhibiting substances that were extracted along with the DNA from the original sample, and that may reduce the efficiency of the polymerase, will be diluted during the second reaction. The overall amplification will be increased, leading to greater sensitivity (although, theoretically, single copies of the target sequence should be detectable with a single PCR). The product from the first reaction will only re-amplify if it is, indeed, the correct product of the same sequence as the original target, which reduces the need for a secondary hybridisation or method to ensure specificity. Furthermore, the product from the second reaction will not be amplified by the first reaction so that carryover contamination may be reduced. However, these advantages are usually overshadowed by the fact that the reaction tube has to be opened half way through the assay and material transferred to a new set of tubes. This increases the likelihood of tube to tube contamination and cannot be performed within the strict segregation of amplified product from reaction tubes described above.

A "single tube nested" PCR has also been described for tuberculosis. In this procedure the two PCRs are separated by the temperature at which the sets of primers will anneal to the target DNA. During the first stage the annealing temperature is high, allowing long outer primers to bind and amplify a specific product, despite the presence in the reaction tube of shorter inner primers. By lowering the annealing temperature, without opening the tube, the shorter primers can then amplify their target within the original product. The longer primers will also re-anneal but, if the short primers are in excess, a specific product will be produced. ${ }^{23}$ This technique has the advantages of sensitivity and specificity of the traditional "nested" PCR, but does not have the benefit of diluting inhibitory substances.

PCR works best with ultrapure reagents and solutions of predetermined concentrations. It is therefore not surprising that DNA extracted from complex body fluids such as sputum should sometimes be contaminated with substances that inhibit the polymerase. Although false negative results are a less common problem than false positives, the addition of target DNA that has been genetically engineered to produce a product of a different size when amplified by the same PCR allows them to be quantified. ${ }^{26}{ }^{27}$ However, whereas the different products can be detected by agarose gel electrophoresis, colorimetric detection methods suitable for use with ELISA plate readers cannot distinguish different sized products..$^{23}$ Furthermore, the modified "control" target may compete with the real target leading to lower sensitivity. ${ }^{26}$

Despite a few small studies that found positive PCRs in patients with asymptomatic tuberculous infection, or previous fully treated disease ${ }^{2829}$ a consensus is now emerging from the thousands of specimens that have been tested. ${ }^{21273031}$ It seems that PCR can indeed detect DNA from clinical samples containing $M$ tuberculosis within a day. Sputum has been the starting point for most of these assays, although a recent study reported that $M$ tuberculosisspecific nucleic acids could also be amplified from the blood of eight patients with smear positive pulmonary tuberculosis. ${ }^{32}$ The sensitivity of most assays is similar to that of culture and the specificity is also high if appropriate care and controls are used. However, direct comparisons with routine mycobacterial culture are of limited value since it is generally accepted that a proportion of patients with "genuine" tuberculosis will have negative cultures. PCR also remains positive for longer than either microscopy or culture once a patient starts treatment, ${ }^{334}$ although more studies are needed to quantify this more accurately. Prospectively designed studies that include close follow up of patients for at least six months after specimens are submitted are needed to define the gold standard against which PCR results should be compared to calculate sensitivity and specificity. Furthermore, these comparisons must be performed in a blinded fashion; it will always be hard to avoid bias when "discrepancy resolution" is undertaken. ${ }^{17}$

The newer amplification techniques, particularly TMA, will reach a similar consensus more rapidly as the trials will be larger and better designed with the experience of PCR to emulate. The challenges for the commercial companies taking these tests into the market place will be to develop closed lysis systems that do not require repeated opening of reaction vessels, and methods to detect the amplified product that do not require the tube to be opened again. However, it must be borne in mind that no test has so far proved to be much more sensitive than culture, and that the use of Bactec (Becton Dickenson) radiospirometric culture with probes commercially available from Genprobe may give a culture result in only a week.

\section{Future prospects}

New methods of diagnosis will inevitably become most available in the parts of the world with least tuberculosis. The emphasis here will be on benefits for the individual patient and resource allocation will be a relatively minor concern. It is also in these environments that new tests will be most challenged. In the setting of low disease prevalence, an extremely high specificity will be required 
to prevent most of the reported positive results being false positives.

In the parts of the world where tuberculosis is still killing millions and control programmes are swamped by the rising tide of HIV-related disease, the role of any new techniques will have to be compared very critically with sputum microscopy which is already capable of detecting those cases most at risk of continuing to spread the epidemic. Nonetheless, the clinical officer is still faced with many symptomatic people with negative sputum smears for whom he must decide how best to care. ${ }^{35}$ Many of the obstacles to using molecular amplification techniques in these situations are being overcome. The reagent cost of the single tube nested PCR test is less than that of a sheet of $x$ ray film. The suggestion that PCR will be oversensitive and detect those with latent tuberculous infection or treated tuberculosis ${ }^{2829}$ is not borne out by work in $\mathrm{Zambia}^{36}$ or Tanzania. ${ }^{34}$ The "high technology" of amplification techniques should be no more of an impediment than the introduction of ELISA technology which is now widespread in much of the world.

Molecular diagnosis of tuberculosis will never replace microscopy as the foundation of tuberculosis control, but health facilities sophisticated enough to produce chest radiographs should follow developments in molecular diagnostics closely.

The ZAMBART molecular laboratory is funded by grants from the Science and Technology for Developing Countries programme of the Commission of the European Communities and the Tuberculosis Unit of the World Health the European Communities and the Tuberculosis Unit
Organisation. PG-F is a Beit Memorial Medical Fellow.

\section{ZAMBART Project,}

\section{P GODFREY-FAUSSETT}

Department of Medicine,

University Teaching Hospital,

PO Box 50110,

Lusaka,

Zambia

1 Lucas SB, Hounnou A, Peacock C, Beaumel A, Djomand G, Ngbichi JM et al. The mortality and pathology of HIV infection in a West African city. AIDS 1993;7:1569-79.

2 Gilks CF, Brindle RJ, Otieno LS, Bhatt SM, Newnham RS, Simani PM, et al. Extrapulmonary and disseminated tuberculosis in HIV-1 seropositive patients presenting to the acute medical services in Nairobi. AIDS 1990; 4:981-5.

3 Mukai Y, Perriens JH, St Louis ME, Brown C, Prignot J, Willame JC, et al. Spectrum of immunodeficiency in HIV-1-infected patients with et al. Spectrum of immunodeficiency in HIV-1-infected

4 Jones BE, Young SMM, Antoniskis D, Davidson PT, Karmer F, Barnes PF. Relationship of the manifestations of tuberculosis to CD4 cell counts in patients with human immunodeficiency virus infection. Am Rev Respir Dis 1993;148:1292-7.

5 Brindle RJ, Nunn PP, Githui W, Allen BW, Gathua S, Waiyaki P. Quantitative bacillary response to treatment in HIV-associated pulmonary tuberculosis. Am Rev Respir Dis 1993;147:958-61.

6 Elliott AM, Luo N, Tembo G, Halwiindi B, Steenbergen G, Machiels L, et al. Impact of HIV on tuberculosis in Zambia: a cross sectional study.BMF 1990;301:412-5.

7 Githui WA, Nunn PP, Juma E, Karimi F, Brindle R, Kamunyi R, et al. Cohort study of HIV infection in tuberculosis patients, Nairobi, Kenya: comparison of bacteriological results. Tuberc Lung Dis 1992;73:203-9.

8 Daley CL, Small PM. Schecter GF, Schoonik G, McAdam R, Jacobs WR, et al. An outbreak of tuberculosis with accelerated progression among persons infected with human immunodeficiency virus. $N$ Engl $\mathcal{f} \mathrm{Med}$ 1992;326:231-5.

9 Elliott AM, Halwiindi B, Hayes RJ, Luo N, Tembo G, Machiels L, et al. The impact of HIV on presentation and diagnosis of TB in a cohort study in Zambia. F Trop Med Hyg 1993;96:1-11.

$10 \mathrm{McFadden} \mathrm{J}$ (ed). Molecular biology of the mycobacteria. London: Academic Press, 1990.
11 Arruda S, Bomfim G, Knights R, Huimabyron T, Riley LW. Cloning of an $M$ tuberculosis DNA fragment associated with entry and survival inside cells. Science 1993;261:1454-7.

12 Pao CC, Lin SS, Wu SY, Juang WM, Chang CH, Lin JY. The detection of mycobacterial DNA sequences in uncultured clinical specimens with

13 Saiki RK, Gelfand DH, Stoffel S, et al. Primer-directed enzymatic amplification DNA with a thermostable DNA polymerase. Science 1988;239: 487-91.

14 Brisson-Noel A, Gicquel B, Lecossier D, Levy-Frebault V, Nassif X, Hance AJ. Rapid diagnosis of tuberculosis by amplification of mycobacterial DNA in clinical samples. Lancet 1989;ii: 1069-71.

15 Winndeen ES, Batt CA, Wiedmann M. Non-radioactive detection of Mycobacterium tuberculosis LCR products in a microtitre plate format. Mol Cell Probe 1993;7:179-86.

16 Jonas V, Alden MJ, Curry JI, Kamasingo K, Knott CA, Langford R, et al. Detection and identification of Mycobacterium tuberculosis directly from sputum sediments by amplification of rRNA. F Clin Microbiol 1993;31: 2410-6.

17 Miller N, Hernandez SG, Cleary TJ. Evaluation of Gen-probe amplified Mycobacterium tuberculosis direct test and PCR for direct detection of Mycobacterium tuberculosis in clinical specimens. $f$ Clin Microbiol 1994;32: 393-7.

18 Walker GT, Little MC, Nadeau JG, Shank DD. Isothermal in vitro amplification of DNA by a restriction enzyme/DNA polymerase system. Proc Natl Acad Sci USA 1992;89:392-6.

19 Aber VR, Allen BW, Mitchison DA, Ayuma P, Edwards EA, Keyes AB. Quality control in tuberculosis bacteriology. 1. Laboratory studies on isolated positive cultures and the efficiency of direct smear examination. Tubercle 1980;61:123-33.

20 Small PM, McClenny NB, Singh SP, Schoolnik GK, Tompkins LS, Mickelsen PA. Molecular strain typing of Mycobacterium tuberculosis to confirm cross-contamination in the mycobacteriology laboratory and modification of procedures to minimise occurrence of false-positive cultures. $f \mathrm{Clin}$ Microbiol 1993;31:1677-82.

21 Noordhoek GT, Kolk AHJ, Bjune G, Catty D, Dale JW, Fine PEM, et al. Sensitivity and specificity of PCR for detection of Mycobacterium tuberculosis - a blind comparison study among seven laboratories. 7 Clin Microbio 1994;32:277-84.

22 Kwok S, Higuchi R. Avoiding false positives with PCR. Nature 1989;339: 237-8.

23 Wilson SM, McNerney R, Nye PM, Godfrey-Faussett P, Stoker NG, Voller A. Progress towards a simplified polymerase chain reaction and its application to the diagnosis of tuberculosis. F Clin Microbiol 1993;31: $776-82$

24 Longo MC, Berninger MS, Hartley JL. The use of uracil DNA glycosylase to control carry-over contamination in polymerase chain reactions. Gene 1990;93:125-8.

25 Pierre C, Lecossier D, Boussougant Y, Bocart D, Joly V, Yeni P, et al. Use of reamplification protocol improves sensitivity of detection of mycobacterium TB in clinical samples by amplification of DNA. $\mathcal{f}$ Clin Microbiol 1991;29:712-7.

26 Andersen AB, Thybo S, Godfrey-Faussett P, Stoker NG. Polymerase chain reaction for detection of Mycobacterium tuberculosis in sputum. Eur $\mathfrak{f}$ Clin Microbiol Infect Dis 1993;12:922-7.

27 Kox LFF, Rhienthong D, Miranda AM, Udomsantisuk N, Ellis K, Vanleeuwen $\mathrm{J}$, et al. More reliable PCR for detection of Mycobacterium tuberculosis in clinical samples. $₹$ Clin Microbiol 1994;32:672-8.

28 Schluger NW, Kinney D, Harkin TJ, Rom WN. Clinical utility of the polymerase chain reaction in the diagnosis of infections due to $\mathrm{Myco}$ bacterium tuberculosis. Chest 1994;105:1116-21.

29 Walker DA, Taylor IK, Mitchell DM, Shaw RJ. Comparison of polymerase chain reaction amplification of two mycobacterial DNA sequences, IS6110 and $65 \mathrm{kDa}$ antigen gene, in the diagnosis of tuberculosis. Thorax 1992; 47:690.

30 Clarridge JE, Shawar RM, Shinnick TM, Plikaytis BB. Large-scale use of polymerase chain reaction for detection of Mycobacterium tuberculosis in a routine mycobacteriology laboratory. $\mathcal{F}$ Clin Microbiol 1993;31:2049-56.

31 Eisenach KD, Sifford MD, Cave MD, Bates JH, Crawford JT. Detection of Mycobacterium tuberculosis in sputum samples using a polymerase chain reaction. Am Rev Respir Dis 1991;144:1160-3.

32 Schluger NW, Condos R, Lewis S, Rom WN. Amplification of DNA of Mycobacterium tuberculosis from peripheral blood of patients with pulmonary tuberculosis. Lancet 1994;344:232-3.

33 Levee $G$, Glaziou P, Gicquel B, Chanteau S. Follow up of tuberculosis patients undergoing standard anti-tuberculosis chemotherapy by using a polymerase chain reaction. Res Microbiol 1994;145:5-8.

34 Kennedy N, Gillespie SH, Saruni AOS, Kisyombe G, McNerney R, Ngowi FI, et al. Polymerase chain reaction for assessing treatment response in patients with pulmonary tuberculosis. $\mathcal{F}$ Infect Dis 1994;170:713-6.

35 Lherminez RH. Urgent need for a new approach to the diagnosis of tuberculosis in developing countries in the decade of AIDS. Trop Geogr Med 1993;45:145-9.

36 Munthali L, Wilson S, Bhat G, Osborne C, Luo N, Kahenya G, et al. Polymerase chain reaction (PCR) for the detection of tuberculosis in Zambia. Presented at Tuberculosis - Towards 2000 Meeting, Pretoria, March 1994. 